\title{
Simultaneous Determination of Urocanic Acid Isomers and Histidine in Human Stratum Corneum by High-Performance Liquid Chromatography
}

\author{
Naohiro Tateda, ${ }^{*}$ Kiichi Matsuhisa, ${ }^{*}$ Kiyoshi Hasebe, ${ }^{* *}$ and Toshiaki MiUra***† \\ *Asahikawa National College of Technology, Shunkodai, 2-2, Asahikawa 071-8142, Japan \\ **Graduate School of Environmental Earth Science, Hokkaido University, \\ Nishi-5, Kita-10, Kita, Sapporo 060-0810, Japan \\ ***College of Medical Technology, Hokkaido University, Nishi-5, Kita-12, Kita, Sapporo 060-0812, Japan
}

(Received September 4, 2000; Accepted March 16, 2001)

\begin{abstract}
Histidine ammonia-lyase (E.C.4.3.1.3) catalyzed degradation to trans-urocanic acid (UCA) is one of the major metabolic pathways of histidine in liver and skin. Trans-UCA is further metabolized in liver by urocanase (E.C.4.2.1.49) while it accumulates in skin, predominantly in the stratum corneum because no urocanase activity is present in the epidermis. ${ }^{1,2}$ Both in vitro and in the skins of experimental animals and humans, trans-UCA isomerizes to cis-UCA following exposure to ultraviolet (UV) light. $^{2-4}$ In addition, topical application of trans-UCA was shown to reduce UV-induced erythema in humans. ${ }^{3,5}$ Therefore, trans-UCA has been believed to function as a natural sun-screen agent against sunburn and against UVinduced damage to DNA in the skin, ${ }^{2,3,5}$ though recent studies have shown that the level of an endogenous trans-UCA in the skin is too low to exert its photoprotective effect. ${ }^{6}$ In contrast to the trans isomer, cis-UCA acts an initiator of immunosuppression which may be a critical step in UV-induced pathogenesis of skin cancer and other cutaneous and systemic diseases. ${ }^{2,47-9}$ Though a variety of factors affect UCA contents in the skin, ${ }^{10,11}$ the major ones are the level of bacterial flora capable of degrading UCA, ${ }^{11}$ and the availability of free histidine produced from the histidine-rich protein of the keratohyalin granule in the epidermis. ${ }^{10}$ Therefore, the assays of UCA isomers as well as histidine are of great importance in the photodermatological study.
\end{abstract}

Geometrical isomers of UCA in the skin have been determined by high-performance liquid chromatography (HPLC) with a UV absorbance detector., ${ }^{42-14}$ However, histidine has also been measured by alternative methods ${ }^{10,15,16}$ such as an amino acid analyzer because no method other than a capillary gas chromatography-mass spectrometry $^{17}$ was available for the simultaneous assay of the three substances. Recently, Takahashi et al. have reported the HPLC method using precolumn derivatization with dabsyl chloride for the assays of UCA isomers and histidine in the skin. ${ }^{18}$ However, the reagent is reactive with all other amino acids and amines in biological samples. Thus, the method requires precise chromatographic separations of UCA isomers and histidine from the interferents and thus becomes time-consuming. On the other hand, we have developed a specific HPLC method for the

\footnotetext{
† To whom correspondence should be addressed.

E-mail: miura@cme.hokudai.ac.jp
}

assay of serum histidine,${ }^{19}$ which is based on the separation by a reversed-phase ion pair chromatography followed by the selective detection of histidine with postcolumn fluorescence derivatization using $o$-phthalaldehyde (OPA). Since its separation mode has also been used for the HPLC assay of UCA isomers, ${ }^{13,14}$ we attempted to develop a reversed-phase ion pair chromatography with the sequential detections of UCA isomers at $264 \mathrm{~nm}$ and histidine with the postcolumn derivatization. This HPLC method was applicable to the simultaneous assay of UCA isomers and histidine in human stratum corneum in combination with the tape-stripping method. ${ }^{14,20,21}$

\section{Experimental}

\section{Chemicals}

Histidine monohydrochloride, agamatine sulfate, spermidine phosphate, putrescine dihydrochloride, glutathione, sodium heptanesulfonate and OPA were obtained from Wako Pure Chemical Industries Ltd. (Osaka). 1-Methylhistidine and 3methylhistidine were purchased from Hoechst Japan Ltd. (Tokyo). Amino acids were from Ajinomoto Company (Tokyo). Pyroglutamic acid and histamine dihydrochloride were obtained from Tokyo Kasei Organic Chemicals (Tokyo). Trans-UCA was obtained from Aldrich (Milwaukee, WI, USA). HPLC-grade acetonitrile was from Kanto Chemical (Tokyo). All other reagents used were of analytical grade. Milli-Q (Nihon Millipore Kogyo, Yonezawa) water was used in the preparations of an eluent and reagent solutions. Stock standard solutions of trans-UCA $(10 \mathrm{mM})$ and histidine $(20 \mathrm{mM})$ were prepared in $0.02 \mathrm{M} \mathrm{HCl}$ and stored in a refrigerator. Working standard solutions of trans-UCA and histidine were prepared by diluting their stock solutions with $0.02 \mathrm{M} \mathrm{HCl}$ before use. A standard solution of a mixture of cis- and trans-UCA was prepared by UV irradiation of $0.2 \mathrm{mM}$ trans-UCA in $0.02 \mathrm{M}$ $\mathrm{HCl}$ according to the method of Shibata et al. ${ }^{14}$ OPA solution was freshly prepared by dissolving in HPLC-grade acetonitrile.

\section{Preparation of human stratum corneum extract}

According to the reported methods ${ }^{14,20,21}$ with slight modification, the stratum corneum was stripped by applying a piece of cellophane adhesive tape $(20 \mathrm{~mm} \times 18 \mathrm{~mm}$ wide; Nichiban, Tokyo) to the skin in the region of the inner forearm, 
the lower abdomen or the back of the hand for $10 \mathrm{~s}$. UCA and histidine on the tape were extracted by immersing the tape in $1.5 \mathrm{ml}$ of $0.1 \mathrm{M} \mathrm{KOH}$ in a $2.0 \mathrm{ml}$ screw-capped polypropylene tube for $30 \mathrm{~min}$ with occasional vortexing. The extract was neutralized with $0.5 \mathrm{ml}$ of $0.38 \mathrm{M} \mathrm{HCl}$ and filtered through a $0.45 \mu \mathrm{m}$ membrane filter (Millipore, Berford, MA, USA). A portion of the filtrate $(20 \mu \mathrm{l})$ was injected onto the HPLC column. The 20 successive tape-stripping experiments were performed on one of the authors and the single tape-stripping experiments were done on the backs of the hands of 10 young female students (range 20-22 years) as volunteers after their informed consents were obtained.

\section{Chromatographic system and conditions}

HPLC system consisted of an L-6000 pump (Hitachi, Tokyo) and an LC-9A pump (Shimadzu, Kyoto) for deliveries of an eluant and OPA reagent, a DGU-12A degasser (Shimadzu), a CTO-10A column oven (Shimadzu), a Rheodyne Model 7725i sample injector (Rheodyne, Cotati, CA, USA), a UV-4200 absorbance detector equipped with a $17.7 \mu$ flow cell (Hitachi), an F-1050 fluorescence detector equipped with a $12 \mu \mathrm{l}$ flow cell, and two D-2500 data processors (Hitachi). Separation was performed at $40^{\circ} \mathrm{C}$ by using a $100 \times 4.6 \mathrm{~mm}$ i.d. Develosil ODS UG-3 column with a particle size of $3 \mu \mathrm{m}$ (Nomura Chemical, Seto) as an analytical column which was protected by a guardpack cartridge column $($ a $10 \times 4.0 \mathrm{~mm}$ i.d. Develosil ODS UG5 , particle size of $5 \mu \mathrm{m})$, and a 7:93 (v/v) mixture of acetonitrile and $20 \mathrm{mM}$ potassium phosphate buffer ( $\mathrm{pH}$ 3.7) containing 5 $\mathrm{mM}$ sodium heptanesulfonate as an eluent. OPA reagent used for the postcolumn detection of histidine was a 15:1 (v/v) mixture of $50 \mathrm{mM}$ potassium phosphate buffer $(\mathrm{pH} \mathrm{8.0)}$ ) and 50 $\mathrm{mM}$ OPA in acetonitrile. Both the eluent and OPA reagent were filtered through the membrane filter before use. The eluent was delivered to the column at a flow-rate of $0.5 \mathrm{ml} \mathrm{min} \mathrm{m}^{-1}$ through a preheater tube (stainless-steel tube, $10 \mathrm{~m} \times 0.8 \mathrm{~mm}$ i.d.) and the sample solution $(20 \mu \mathrm{l})$ was introduced to the column. The eluate from the column was passed through a UV detector and was then added with OPA reagent delivered at a flow-rate of $0.5 \mathrm{ml} \mathrm{min}^{-1}$ to a mixing T-joint through a preheater tube (stainless-steel tube, $10 \mathrm{~m} \times 0.8 \mathrm{~mm}$ i.d.) and a resistor polytetrafluoroethylene (PTFE) tube $(20 \mathrm{~m} \times 0.25 \mathrm{~mm}$ i.d.). The mixture was passed through a reactor tube (coiled PTFE tube, $2.5 \mathrm{~m} \times 0.5 \mathrm{~mm}$ i.d., coil diameter of $20 \mathrm{~mm}$ ) to generate fluorescence. All the columns, preheater-, resistor- and reactor tubes were placed in the column oven maintained at $40^{\circ} \mathrm{C}$. UCA isomers were detected at $264 \mathrm{~nm}$, whereas histidine was measured by the fluorescence at $435 \mathrm{~nm}$ with an excitation wavelength of $365 \mathrm{~nm}$ (uncorrected). Elutions of other standard biological substances undetected by either of the above methods were monitored at $220 \mathrm{~nm}$, except for polyamines such as agmatine, putrescine and spermidine which were detected by a postcolumn derivatization with a mixture of OPA and 2mercaptoethanol, a fluorogenic reagent system for primary amines. $^{22}$

\section{Results and Discussion}

HPLC method for the simultaneous determinations of UCA isomers and histidine

Among a number of HPLC conditions available for the separation of UCA isomers, ${ }^{4,12-14}$ we chose a reversed-phase ion pair chromatography because its separation mode was also successfully used in our HPLC method for the assay of histidine. ${ }^{19}$ As a result, separation of UCA isomers and

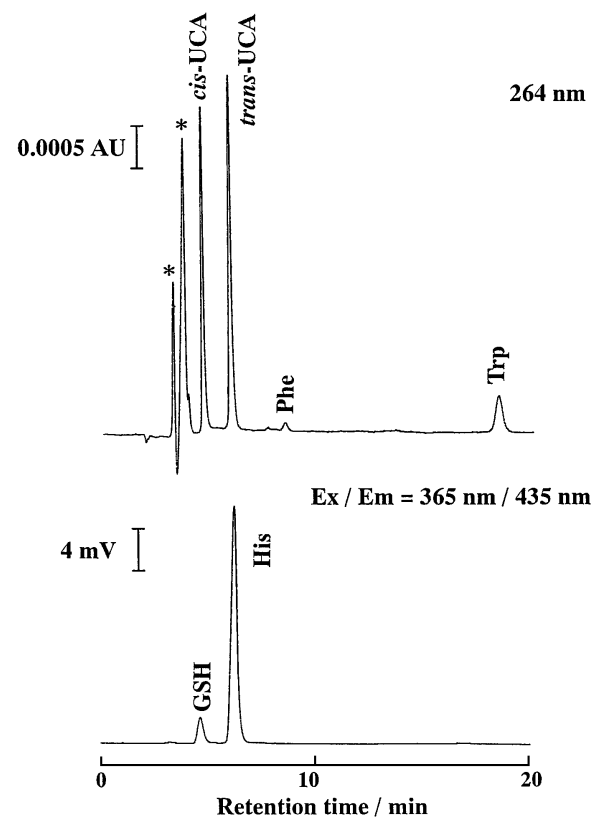

Fig. 1 Chromatograms monitored with UV- and fluorescence detectors of a mixture of cis-urocanic acid (cis-UCA), trans-urocanic acid (trans-UCA), 20 amino acids, glutathione (GSH) and pyroglutamic acid. Injected amount of each was 20 pmol per $20 \mu \mathrm{l}$. His, histidine; Phe, phenylalanine; Trp, tryptophane. *Solvent peak. Ex, excitation wavelength; Em, emission wavelength.

histidine was achieved at $40^{\circ} \mathrm{C}$ on a $\mathrm{C} 18$ reversed phase column with a 7:93 (v/v) mixture of acetonitrile and $20 \mathrm{mM}$ phosphate buffer ( $\mathrm{pH}$ 3.7) containing $5 \mathrm{mM}$ sodium heptanesulfonate at a flow rate of $0.5 \mathrm{ml} \mathrm{min}{ }^{-1}$. After the separation, UV $(264 \mathrm{~nm})$ detector and the postcolumn derivatization with OPA were used for the sequential detections of UCA isomers and histidine. The postcolumn derivatization was carried out under the same conditions as our original ones ${ }^{19}$ except that acetonitrile was used instead of methanol as a solvent for OPA.

Figure 1 shows chromatograms of a standard mixture of 20 amino acids, cis- and trans-UCA, glutathione and pyroglutamic acid, the last one is known to present in the skin. In the chromatogram monitored at $264 \mathrm{~nm}$, cis- and trans-UCA were observed at 4.9 and $6.4 \mathrm{~min}$, respectively, which were well separated from all detectable amino acids. Histidine was shown to be eluted at $5.5 \mathrm{~min}$ by monitoring at $220 \mathrm{~nm}$ but it was not detected at $264 \mathrm{~nm}$. On the other hand, histidine was detected at 6.2 min with a fluorescence detector. The retention time (Rt) was slightly longer than that $(5.5 \mathrm{~min})$ described above because the reaction coil was inserted in the line. Though glutathione was also detected at $3.7 \mathrm{~min}$, the other 19 amino acids (Rt $<19$ $\mathrm{min})$, pyroglutamic acid $(\mathrm{Rt}=2.3 \mathrm{~min})$, ornithine $(\mathrm{Rt}=5.4$ $\mathrm{min})$, 1-methylhistidine $(\mathrm{Rt}=5.5 \mathrm{~min})$, lysine $(\mathrm{Rt}=5.6 \mathrm{~min})$ and 3-methylhistidine ( $\mathrm{Rt}=5.8 \mathrm{~min})$ gave no peak and thus did not interfere with the quantitative detection of histidine. No interference was also observed from histamine $(\mathrm{Rt}=39.7 \mathrm{~min})$ and polyamines (Rt $>32 \mathrm{~min}$ )

The present HPLC method gave linear calibration curves for cis-UCA $(r=0.999)$ and trans-UCA $(r=0.999)$ over the range of $0.5-1500$ pmol per injection $(20 \mu \mathrm{l})$ with the relative standard deviation (RSD) of $0.58 \%$ and $0.31 \%$ at $10 \mathrm{pmol}(n=$ $10)$, and with the detection limits $(S / N=5)$ of $40 \mathrm{fmol}$ and 70 fmol, respectively. For histidine, a linear calibration curve $(r=$ 0.999 ) was obtained over the range of $0.1-1000$ pmol per 

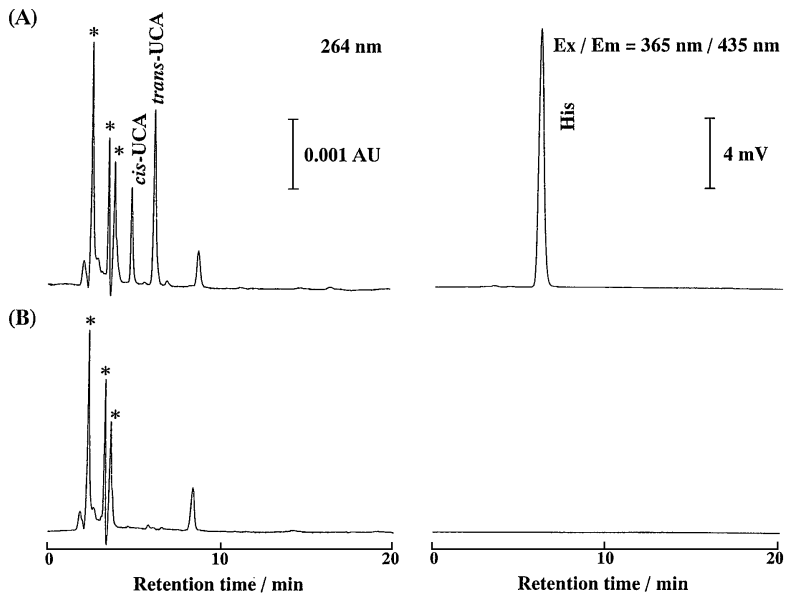

Fig. 2 Chromatograms monitored with UV- and fluorescence detectors of the extract of human stratum corneum stripped from the skin on the back of the hand (A) and the blank extract from a piece of cellophane adhesive tape (B). Detected amounts were 9.7, 7.0 and 11.7 pmol per injection $(20 \mu \mathrm{l})$ for cis-urocanic acid (cis-UCA), trans-urocanic acid (trans-UCA) and histidine (His), respectively. "Solvent peak. Ex, excitation wavelength; Em, emission wavelength.

injection $(20 \mu \mathrm{l})$ with RSD of $0.9 \%$ at $10 \mathrm{pmol}(n=10)$ and with the detection limit $(S / N=5)$ of $10 \mathrm{fmol}$.

In order to apply the HPLC method to the simultaneous assays of UCA isomers and histidine in human stratum corneum, we chose the tape-stripping method as a simple and non-invasive sampling procedure. . $^{140,21}$ Figure $2 \mathrm{~A}$ shows the typical chromatograms of an extract from the stratum corneum of the back of the hand. Both the chromatograms monitored with UVand fluorescence detectors were very simple and UCA isomers and histidine were clearly detected. In the chromatogram monitored at $264 \mathrm{~nm}$, however, a small peak due to tryptophane at $18.8 \mathrm{~min}$ was observed in the several stratum corneum extracts. Thus, at least $20 \mathrm{~min}$ is required for one run of HPLC analysis. Figure 2B shows the chromatograms of blank extract from the tape, where neither UCA isomers nor histidine were detected. Though several unknown substances (not amino acids) were observed in the chromatogram monitored at 264 $\mathrm{nm}$, they did not interfere with the assay of UCA isomers.

Extraction efficiencies of UCA isomers and histidine with 0.1 $\mathrm{M} \mathrm{KOH}$ from the stripped stratum corneum on the cellophane tape were confirmed by repeating the extraction procedure on the previously extracted tape. The mean percentages $(n=20)$ of the second extracted amount in the sum of the first and second extracted amounts were $1.8 \%$ for a total of UCA and $1.3 \%$ for histidine, indicating that both substances were recovered quantitatively from the tape with the first extraction.

The reproducibility of the present method was also examined by using the pooled extract from the tape-stripped stratum corneum on the back of the hand. The RSD of within assay were $2.3,0.6$ and $0.6 \%(n=10)$ and those of day-to-day assay were $2.0,1.1$ and $1.6 \%(n=6)$ for cis-UCA, trans-UCA and histidine, respectively. Norval et al. claimed poor reproducibility of a reversed-phase ion pair chromatography for the assays of UCA isomers. ${ }^{23}$ However, our HPLC method with the same separation mode showed good reproducibilities in their retention times and peak heights. These improvements are probably due to precise temperature control $\left(40^{\circ} \mathrm{C}\right)$ of the eluent by insertion of a preheater coil in the line, as described in Experimental, such a coil is inherently required for the
Table 1 Contents of urocanic acid isomers and histidine in the single tape-stripped human stratum corneum from the back of the hand measured in December

\begin{tabular}{|c|c|c|c|c|c|c|}
\hline \multirow{3}{*}{ Volunteer } & \multicolumn{4}{|c|}{ Content $/ \mathrm{nmol} \mathrm{cm}^{-2}$} & \multirow{3}{*}{$C i s^{\mathrm{b}}, \%$} & \multirow{3}{*}{$\mathrm{UCA}^{\mathrm{a}} / \mathrm{His}^{\mathrm{a}}$} \\
\hline & \multicolumn{3}{|c|}{$\mathrm{UCA}^{\mathrm{a}}$} & \multirow[t]{2}{*}{$\mathrm{His}^{\mathrm{a}}$} & & \\
\hline & Cis & Trans & Total & & & \\
\hline A & 0.097 & 1.062 & 1.159 & 1.275 & 8.4 & 0.91 \\
\hline B & 0.051 & 0.211 & 0.262 & 0.280 & 19.5 & 0.93 \\
\hline $\mathrm{C}$ & 0.011 & 0.061 & 0.072 & 0.078 & 15.3 & 0.92 \\
\hline D & 0.028 & 0.135 & 0.163 & 0.616 & 17.2 & 0.26 \\
\hline E & 0.195 & 0.270 & 0.465 & 0.325 & 41.9 & 1.43 \\
\hline F & 0.041 & 0.163 & 0.204 & 0.234 & 20.1 & 0.87 \\
\hline G & 0.123 & 0.333 & 0.456 & 0.638 & 27.0 & 0.71 \\
\hline $\mathrm{H}$ & 0.027 & 0.191 & 0.218 & 0.160 & 12.4 & 1.36 \\
\hline I & 0.089 & 0.341 & 0.430 & 0.418 & 20.7 & 1.03 \\
\hline $\mathrm{J}$ & 0.067 & 0.118 & 0.185 & 0.258 & 36.2 & 0.72 \\
\hline Mean & 0.073 & 0.289 & 0.361 & 0.428 & 21.9 & 0.91 \\
\hline SD & 0.055 & 0.287 & 0.311 & 0.348 & 10.4 & 0.33 \\
\hline
\end{tabular}

a. UCA and His represent urocanic acid and histidine, respectively. b. The percentage of UCA presents as cis-isomer.

reproducible postcolumn detection of histidine with OPA. ${ }^{19}$

\section{Distribution of UCA isomers and histidine in human stratum corneum}

Since the present method enabled the sensitive and reliable assays of UCA isomers and histidine in the stratum corneum, their distribution patterns were investigated in the stratum corneum of several body sites.

Table 1 shows the concentrations of UCA isomers and histidine in the single tape-stripped stratum corneums from the back of the hand of 10 healthy female volunteers. The interindividual variations were large in the levels of UCA isomers and histidine and in the percentage of cis-UCA, whereas the variation was less in the ratio of total UCA to histidine.

Figure 3 shows the results of the successive tape-stripping experiments where twenty strips were successively taken from the same location of the inner forearm, the back of the hand and the lower abdomen of the same subject. The levels of UCA isomers varied depending on the number of strips as well as body site. The level of histidine also changed with the number of strips, however, its change closely paralleled that of a total UCA in all cases. Schwarz et al. ${ }^{15}$ who measured histidine by amino acid analyzer, briefly mentioned the similar tendency between UCA and histidine on the skin of the left scapula.

Figure 4 shows the percentage of UCA present as cis-isomer. The percentages measured in October were in the order of the back of the hand > the inner forearm > the lower abdomen. This order is reasonable because the trans-cis isomerization of UCA occurs more easily in a sunlight-exposed site (the back of the hand) than in a less exposed site (the inner forearm) or in a shielded site (the lower abdomen). ${ }^{6,14,24}$ In the case of the inner forearm, the cis percentages were higher in July than in October and gradually decreased with the increase in number of strips. The former is explained as seasonal variation in UV radiation dose from the $\operatorname{sun}^{25}$ and the latter is interpreted by the suggestion that the effect of UV radiation gradually decreases from the upper layer to a deeper layer in the skin. ${ }^{21}$

In combination with the tape-stripping method, the present HPLC method is applicable to the simultaneous assays of UCA isomers and histidine in the stratum corneum on the skin of any body site. It has several advantages over the reported 

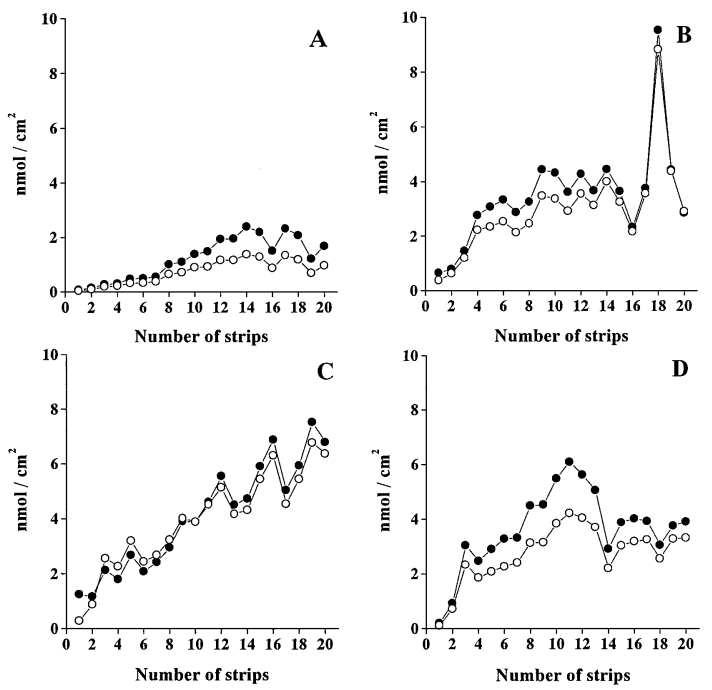

Fig. 3 Distribution patterns of total urocanic acid (•) and histidine ( $\mathrm{O}$ ) in different layers of 20 successive tape-stripped human stratum corneums from skin on the back of the hand (A), the lower abdomen (B) and the inner forearm (D) measured in October, and from the inner forearm (C) measured in July.

methods ${ }^{17,18}$ such as selectivity, simplicity, rapidity, high sensitivity and reproducibility. The present method is also useful in the diagnosis of histidinaemia, one of hereditary metabolic disorders characterized by a virtual deficiency of histidine ammonia-lyase, in which UCA decreases ${ }^{26}$ and histidine probably increases in the stratum corneum.

\section{Acknowledgements}

We thank the students of the College of Medical Technology of Hokkaido University for participating in the single tapestripping experiments as volunteers.

\section{References}

1. A. H. Mehler and H. Tabor, J. Biol. Chem., 1953, 201, 775.

2. H. Morrison, Photodermatology, 1985, 2, 158.

3. H. P. Baden and M. A. Pathak, J. Invest. Dermatol., 1967, 48, 11.

4. M. Norval, T. J. Simpson, and J. A. Ross, Photochem. Photobiol., 1989, 50, 267.

5. O. Fertek, A. Zenisek, and I. M. Hais, Parfümerie Kosmetik, 1969, 50, 223.

6. F. de Fine. Olivarius, H. C. Wulf, P. Therkildsen, T. Poulsen, J. Crosby, and M. Norval, Arch. Dermatol. Res., 1997, 289, 501.

7. F. P. Noonan and E. C. De Fabo, Immunol. Today, 1992, 13, 250.

8. M. Norval, N. K. Gibbs, and J. Gilmour, Photochem. Photobiol., 1995, 62, 209.

9. A. A. El-Ghorr and M. Norval, Photochem. Photobiol., 1997, 65, 866 .

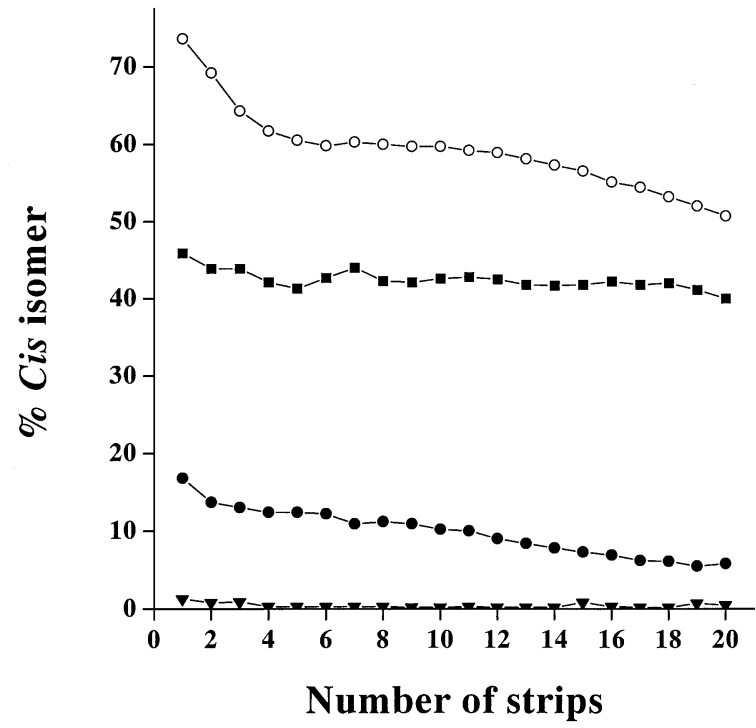

Fig. 4 The percentage of urocanic acid present as cis-isomer in different layers of 20 successive tape-stripped human stratum corneums from skin on the back of the hand $(\boldsymbol{\square})$, the lower abdomen $(\boldsymbol{\nabla})$ and the inner forearm $(\bullet)$ measured in October, and from the inner forearm ( $\mathrm{O}$ ) measured in July.

10. I. R. Scott, Biochem. J., 1981, 194, 829.

11. D. H. Hug, D. D. Dunkerson, and J. K. Hunter, J. Photochem. Photobiol. B: Biol., 1999, 50, 66.

12. J. C. Caron, B. Martin, and B. Shroot, J. Chromatogr., 1982, 230, 125.

13. W. Schwarz, K. Langer, and A. Haag, J. Chromatogr., 1984, 310, 188.

14. K. Shibata, Y. Nishioka, T. Kawada, T. Fushiki, and E. Sugimoto, J. Cromatogr. B, 1997, 695, 434.

15. W. Schwarz, K. Langer, H. Schell, and A. Schönberger, Photodermatology, 1986, 3, 239.

16. L. Juhlin, B. Shroot, B. Martin, and J. C. Caron, Acta Derm. Venereol. (Stockholm), 1986, 66, 295.

17. T. Furuta, M. Katayama, H. Shibasaki, and Y. Kasuya, J. Chromatogr. B, 1992, 576, 213.

18. M. Takahashi and T. Tezuka, J. Chromatogr. B, 1997, 688, 197.

19. N. Tateda, K. Matsuhisa, K. Hasebe, N. Kitajima, and T. Miura, J. Chromatogr. B, 1998, 718, 235.

20. W. Schwarz, J. Chromatogr., 1985, 342, 247.

21. M. Norval, T. J. Simpson, E. Bardshiri, and J. Crosby, Photodermatology, 1989, 6, 142.

22. J. R. Benson and P. E. Hare, Proc. Nat. Acad. Sci. USA, 1975, 72, 619.

23. M. Norval, C. R. McIntyre, T. J. Simpson, S. E. M. Howie, and E. Bardshiri, Photodermatology, 1988, 5, 179.

24. G. Kavanagh, J. Crosby, and M. Norval, Bri. J. Dermatol., 1995, 133, 728.

25. F. de Fine. Olivarius, H. C. Wulf, J. Crosby, and M. Norval, Photochem. Photobiol., 1997, 66, 119.

26. S. Yokoya, E. Tokuhiro, S. Suwa, and H. Maesaka, Eur. J. Pediatr., 1983, 140, 330. 